\title{
Wavelength-Multiplexed Spectrometer Based on Silicon Nanowire Photodetector Array
}

\author{
Jiajun Meng ${ }^{1}$, Shiqiang $\mathrm{Li}^{1}$ and Kenneth B. Crozier ${ }^{1,2^{*}}$ \\ ${ }^{1}$ Department of Electrical and Electronic Engineering, University of Melbourne, Victoria 3010, Australia \\ ${ }^{2}$ School of Physics, University of Melbourne, Victoria 3010, Australia \\ *Corresponding Author e-mail address: kenneth.crozier@unimelb.edu.au
}

It was recently demonstrated that by adding a filter array to photodetector array, a very compact wavelengthmultiplexed spectrometer can be achieved [1,2]. These previous efforts have had both strengths and weaknesses, however. In [1], the filter array comprised etalons with different thicknesses fabricated by a multi-step process. In [2], the filter array comprised quantum dots spotted onto the device with a pipette. Here, we study a spectrometer comprising an array of silicon nanowire (Si NW) photodetectors, formed above an array of planar photodetectors. This device would have manufacturing advantages, being based on Si technology and with all NWs defined in a single lithography step. The NWs would act as photodetectors (converting absorbed light to photocurrent) but would also filter the light transmitted onto the planar photodetectors. After measurement of the photodetector currents, the spectrum of light impinging on the device would be reconstructed numerically.

This approach is based on our demonstration of a device comprising two vertically-stacked PIN photodetectors [3], each of which can be separately addressed electrically (Fig. 1(a)). The top photodetector is a square array of vertical Si NWs. These have responsivity spectra that exhibit peaks whose positions can be tuned across the visible-to-near-infrared range by changing the radii of the NWs. This comes from the NWs acting as waveguides, which leads to wavelength-selective coupling and absorption. As before [3], we here consider NWs containing $\mathrm{p}^{+}$, intrinsic and $\mathrm{n}^{+}$regions with thicknesses of $0.2 \mu \mathrm{m}, 2 \mu \mathrm{m}$ and $0.4 \mu \mathrm{m}$, respectively. The NWs are surrounded by polymethylmethacylate (PMMA) and a $0.2 \mu \mathrm{m}$ layer of indium tin oxide (ITO) is formed on their tops for electrical contact. The bottom photodetector is planar, comprising $\mathrm{n}^{+}$and intrinsic regions with thicknesses of $0.6 \mu \mathrm{m}$ and $4 \mu \mathrm{m}$ respectively, on a $\mathrm{p}^{+}$substrate.

We use finite difference time domain (FDTD) method to simulate structures with different Si NW radii $(R)$ and periods $(P)$. For each structure, we find the power absorption spectra of the intrinsic regions of the NWs and of the planar detector. In Fig. 1b, we plot results for four selected structures. We next study how photocurrents measured from an array of $N$ photodetectors $(N / 2 \mathrm{NW}$ photodetectors and $N / 2$ planar detectors) would be used for spectral reconstruction of the incident light. The photocurrent from detector $k$ is $I_{k}=\int A_{k}(\lambda) P(\lambda) \mathbf{d} \lambda, k=$ $1,2, \ldots, N$, where $A_{k}(\lambda)$ denotes the power absorption spectrum of photodetector $k$ (found by FDTD) and $P(\lambda)$ is the power spectrum of the incident light. We assume that measurement noise is Gaussian and formulate a minimization problem. To test our approach, we take 20 fluorescence spectra from common dyes, as tabulated at [4]. We use the recursive least square (RLS) [5] algorithm to numerically reconstruct the spectra based on the simulated measurements. A typical result (Alexa Fluor) with an assumed measurement signal-to-noise ratio of $S N R_{m}=10 \mathrm{~dB}$ is shown as Fig. 1c. It can be seen that the reconstruction performance is greatly improved when the number of photodetectors is increased from $N=32$ to 128 (i.e. $8 \times 8$ array of NW and planar devices). Indeed, for the 20 test spectra, the error standard deviation reduces from $4-15 \%(N=32)$ to $2-4 \%(N=128)$.

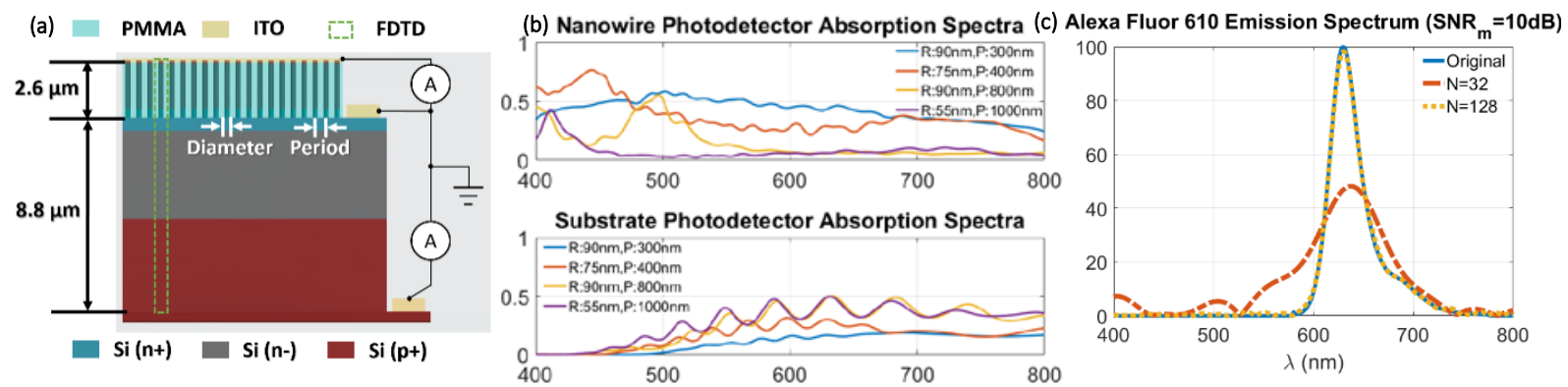

Figure 1. (a) Device schematic. (b) Power absorption spectra of 4 selected NW \& planar photodetector pairs. (c) Comparison between original $\&$ reconstructed emission spectra (normalized) of Alexa Fluor 610 using a $4 \times 4$-pixel $(\mathrm{N}=32$ ) array and $8 \times 8$-pixel $(\mathrm{N}=128)$ array.

\section{References}

[1] E. Huang, Q. Ma and Z. Liu, "Spectrum Engineering for Reconstructive Spectrometry," in Conference on Lasers and Electro-Optics, San Jose, 2016.

[2] J. Bao and M. G. Bawendi, "A colloidal quantum dot spectrometer," Nature, vol. 523, pp. 67-70, 2015.

[3] H. Park and K. B. Crozier, "Vertically Stacked Photodetector Devices Containing Silicon Nanowires with Engineered Absorption Spectra," ACS Photonics, vol. 2, no. 4, pp. 544-549, 2015.

[4] "Fluorescence SpectraViewer | Thermo Fisher Scientific," Thermo Fisher Scientific Inc., 2016. [Online]. Available: http://www.thermofisher.com/au/en/home/life-science/cell-analysis/labeling-chemistry/fluorescence-spectraviewer.html.

[5] M. H. Hayes, "9.4: Recursive Least Squares," in Statistical Digital Signal Processing and Modeling, Wiley, 1996, p. 541. 